Rabaska

Revue d'ethnologie de l'Amérique française

\title{
Musée de la mémoire vivante
}

\section{Judith Douville}

Volume 16, 2018

URI : https://id.erudit.org/iderudit/1051366ar

DOI : https://doi.org/10.7202/1051366ar

Aller au sommaire du numéro

Éditeur(s)

Société québécoise d'ethnologie

ISSN

1703-7433 (imprimé)

1916-7350 (numérique)

Découvrir la revue

Citer ce document

Douville, J. (2018). Musée de la mémoire vivante. Rabaska, 16, 329-331.

https://doi.org/10.7202/1051366ar

Ce document est protégé par la loi sur le droit d'auteur. L'utilisation des services d'Érudit (y compris la reproduction) est assujettie à sa politique d'utilisation que vous pouvez consulter en ligne.

https://apropos.erudit.org/fr/usagers/politique-dutilisation/
Cet article est diffusé et préservé par Érudit.

Érudit est un consortium interuniversitaire sans but lucratif composé de l'Université de Montréal, l'Université Laval et l'Université du Québec à Montréal. Il a pour mission la promotion et la valorisation de la recherche. https://www.erudit.org/fr/ 


\section{QUÉBEC}

Musée de la mémoire vivante

710, avenue de Gaspé Ouest

Saint-Jean-Port-Joli (Québec)

G0R 3G0
Téléphone : (418) 358-0518

Courriel : information@memoirevivante.org

Toile : www.memoirevivante.org

Le Musée de la mémoire vivante est voué à la personne. Les gens s'y racontent afin de partager leurs savoirs et leurs savoir-faire dans un but de sauvegarde et de transmission intergénérationnelle. Il est un musée de société engagé dans la collectivité pour la pérennité de notre patrimoine culturel.

\section{Dix années sont passées}

Le 25 juin 2008, le Musée de la mémoire vivante ouvrait pour la première fois ses portes. Un premier pari était gagné, soit la reconstruction selon l'aspect extérieur et la même volumétrie du manoir des Aubert de Gaspé rasé par les flammes 99 ans plus tôt. Deux défis restaient à relever. Le premier étant d'initier les publics au patrimoine immatériel transmis par l'oralité. Un patrimoine dont la définition était peu connue de la majorité des visiteurs du Musée il y a une décennie. Le second défi était de susciter l'intérêt de ses publics par la mise en musée de l'oralité. Cette diffusion devant interpeller les visiteurs de tout âge, les instruire et les divertir tout en les appelant à participer aux expositions par le partage de leurs propres savoirs. Nous pouvons affirmer que ce but est également atteint. Au cours de ces dix années, le Musée s'est taillé une place enviable à l'échelle provinciale et au sein de la communauté muséale. Sa raison d'être est devenue une évidence.

\section{La collection}

La collection du Musée s'enrichit de témoignages captés par sa propre équipe d'enquêteurs, mais également par des partenaires de diverses régions du Québec. Elle constitue une banque de données cataloguées devenant, avec le temps, notre mémoire collective. Plusieurs témoignages font état de faits et de pratiques qui, pour certains, tomberaient dans l'oubli lors du décès de ses détenteurs. Comptant plus de 2500 témoignages, la collection du Musée est à la base des expositions qui y sont présentées.

Dans un souci de conservation et de diffusion des éléments de sa collection immatérielle, le Musée a procédé à la mise à niveau de son parc informatique au cours de la dernière année.

Le collectionnement lié à des demandes faites par d'autres musées a triplé cette année. Dans ces cas, ce sont des sujets précis que le Musée est appelé à documenter tant par le contenu de sa collection que par de nouvelles enquêtes qui rejoindront cette collection. Le nombre de chercheurs universitaires venant consulter les témoignages a littéralement explosé. Les sujets recherchés sont liés à des projets de mémoires ou de thèses d'étudiants de l'Université Laval, de l'Université de Montréal, de l'Université de Sherbrooke, des Universités du Québec à Montréal et à Rimouski. 
La collection du Musée compte également des artéfacts matériels. Ceux-ci sont en lien avec ses expositions ou avec Philippe Aubert de Gaspé. En 2018, la famille Philippe de Gaspé Beaubien a fait don au Musée d'une reproduction exceptionnelle du manuscrit du roman Les Anciens Canadiens par Philippe Aubert de Gaspé. Il s'agit d'un volume d'une centaine de pages reliées plein cuir et représentant chacune les feuilles écrites par l'auteur et constituant son roman.

\section{Les expositions}

L'exposition Souvenirs de table, après une présence de 10 ans au Musée, a été remplacée brièvement par Hockey du Musée canadien de l'histoire. Afin d'arrimer celle-ci à notre mission, des entrevues ont été intégrées au matériel de base de l'exposition itinérante, selon les thématiques présentées. Dès la mi-septembre, une nouvelle exposition permanente intimement liée au patrimoine immatériel lui a succédé : Excusez-la! (Re) Découvrir la chanson traditionnelle. Entièrement réalisée par le Musée de la mémoire vivante, cette exposition propose des chansons et des souvenirs s'entremêlant pour faire découvrir aux visiteurs la richesse et le dynamisme de ce patrimoine oral porté par des générations.

Août 2018 marque la présence sur la toile de l'exposition virtuelle La Pêche à l'anguille sur la Côte-du-Sud hébergée par le Musée virtuel du Canada, dans le cadre d'Histoires de chez nous. Également réalisée par l'équipe du Musée, cette exposition a connu un volet en salle au Musée québécois de l'agriculture et de l'alimentation et est source de nombreuses demandes de conférences.

\section{Complémentarité et partenariat}

Comme mentionné précédemment, nous sommes partenaires de plusieurs musées et organismes ayant fait appel à nos services pour l'intégration de témoignages dans leurs expositions. Citons, entre autres, le Musée Laurier, le Musée de la culture pop de Trois-Rivières, le Musée minéralogique et minier de Thetford-Mines et la Société d'histoire de la Rivière-du-Nord.

Une finissante en histoire et pédagogie de l'Université Laval, $\mathrm{M}^{\mathrm{me}}$ Marie-Pierre Doyon, a effectué au musée un stage soutenu par Patrimoine Canada pour une carrière vouée au patrimoine. $\mathrm{M}^{\mathrm{me}}$ Doyon a entièrement revu le programme éducatif du Musée. Deux activités sont depuis offertes de manière virtuelle aux élèves des écoles primaires et secondaires. La Commission scolaire de la région de Montmagny a accepté de collaborer à la mise en place de ces programmes en participant expérimentalement aux premiers essais.

\section{Perspectives d'avenir}

Dans la foulée du développement durable et de la convention de l'UNESCO sur le patrimoine immatériel, un projet majeur sera réalisé en 2018-2019 sur le site du Musée. Tout l'aménagement du site a été planifié afin d'atteindre l'un de ses objectifs : soit de souligner la profonde interdépendance entre les patrimoines culturels et naturels dans le but de raviver les souvenirs des visiteurs et de les inviter à partager leur expérience. Une tour donnant accès au belvédère existant suggérera aux visiteurs l'écoute de témoignages de bâtisseurs et d'innovateurs par l'entremise de leur 
téléphone intelligent. Ils pourront également consulter des témoignages sur la vie maritime et le fleuve, la vie agricole. En fait, sur ce qui marque le paysage s'offrant à leur regard.

Judith DouviLLE

\section{Le Centre de conservation du Québec}

Ministère de la Culture et des communications 1825 , rue Semple Québec (Québec) G1N 4B7

Au cours de la période 2017-2018, les actions de conservation du patrimoine menées par l'ensemble des professionnels du Centre de conservation du Québec ont visé à augmenter les services en restauration et en conservation préventive ayant des effets majeurs sur la clientèle et la population, et à contribuer à l'atteinte de plusieurs des objectifs inscrits dans la planification stratégique du ministère de la Culture et des communications.

Tout au long de cette année, plusieurs interventions de la part des spécialistes ont soit permis le sauvetage matériel de biens patrimoniaux, soit contribué à une meilleure connaissance de leur histoire, de leur composition, soit révélé leur valeur patrimoniale.

\section{Contribution aux projets ministériels}

Visant la mise en valeur du patrimoine culturel dans les milieux locaux, le Centre a offert des services en expertise-conseil et en expertise-diagnostic dans le domaine de l'archéologie, de la muséologie du patrimoine religieux et du patrimoine bâti. Par exemple, l'expertise des restaurateurs a été sollicitée dans le cadre de la restauration de bâtiments classés et d'un pont patrimonial. Le travail de documentation par l'étude des composantes intérieures et extérieures (matériaux, techniques de fabrication, méthodes contemporaines d'intervention) et par des sondages et relevés stratigraphiques de finis a contribué significativement à orienter adéquatement les travaux à réaliser. Avant que des travaux de construction sur la structure du bâtiment sous-jacente aient lieu, les boiseries cossues d'une demeure classée ont été démontées puis remises en place de façon méthodique et sécuritaire. Ces actions ont permis leur sauvegarde.

La diffusion de contenus sur les médias sociaux ainsi que la production d'une vidéo sur l'entretien et la restauration de la fontaine de Tourny à Québec ont contribué à la documentation des activités d'actualité et des enjeux existant dans le domaine de la préservation du patrimoine.

Plusieurs formations, dont une sur la pierre, ont été offertes aux intervenants en patrimoine tels que les architectes. Deux conférences professionnelles ont été proposées dans le cadre des Rendez-vous Maestria du Conseil des métiers d'art du Québec en mars 2018. 